SU-ITP-99-26

hep-ph/9905577

January 1, 2019

\title{
Mirage Gauge Coupling Unification and TeV Scale Strings
}

\author{
EDI HALYO ${ }^{*}$ \\ Department of Physics \\ Stanford University \\ Stanford, CA 94305
}

\begin{abstract}
We consider gauge coupling unification in models with TeV scale strings and large compact dimensions realized as type IIB string orientifolds. Following an observation by Ibanez we show that the gauge couplings at low energies can behave as if they effectively unify at $M_{U} \sim 2 \times 10^{16} \mathrm{GeV}$ with $\alpha_{U} \sim 1 / 24$. This requires the $\sigma$ model anomaly coefficients $b_{a}^{i \prime}$ not to be all equal and their ratio to the $\beta$-functions of minimally supersymmetric Standard Model $\beta_{a}$ to be a constant independent of the gauge group. If, in addition, $b_{a}^{i \prime}$ have a gauge group independent constant piece the relation between the unified gauge coupling and the dilaton VEV is modified so that there can be weakly coupled gauge theories arising from strongly coupled strings.
\end{abstract}

\footnotetext{
* e-mail address: halyo@dormouse.stanford.edu
} 


\section{Introduction}

Lately there has been great interest in scenarios involving $\mathrm{TeV}$ scale strings compactified on two or more large dimensions $[1,2]$. In these models the Standard Model degrees of freedom are confined to the world-volume of D-branes. Gravity propagates only in the bulk and its weakness compared to other interactions is a result of the large compact dimensions

$$
M_{P}^{2}=\frac{8 M_{s}^{8} R^{6}}{g_{s}^{2}}
$$

Here $g_{s}$ and $M_{s}$ are the string coupling and string scale respectively and $R^{6}$ is the generic volume of the compact dimensions. Surprisingly, this scenario cannot be easily ruled out by precision and acceleretor experiments or astrophysical observations[3]. Implications of this scenario for particle phenomenology, astrophysics and cosmology have been investigated in some detail[4,5,6]. The strongest bound on the higher dimensional Planck scale, $M_{d+4}$ comes from the supernova 1987A data and is $M_{6}>50 \mathrm{TeV}$ for two large dimensions and $M_{d+4}>1 \mathrm{TeV}$ for more than two large dimensions[7]. In this letter, for simplicity, we take the bound on the higher dimensional Planck scale to hold over the string scale $M_{s}$.

On the other hand, one of the most attractive results of minimally supersymmetric Standard Model with the assumption of a desert up to very high scales is the unification of the gauge couplings with $\alpha_{U}=g_{U}^{2} / 4 \pi \sim 1 / 24$ at $M_{U} \sim 2 \times 10^{16} \mathrm{GeV}$. In models with $\mathrm{TeV}$ scale strings, around $M_{s}$ we find a plethora of new states such as excited string modes, Kaluza-Klein modes, winding modes etc. Moreover field theory is not applicable at this scale and one should use fully-fledged string theory. Therefore unification of gauge couplings at very high energy scales such as $M_{U}$ would seem to be completely accidental. Some aspects of gauge coupling unification in the framework of $\mathrm{TeV}$ scale strings and large compact dimensions have been investigated in [8]. 
Recently Ibanez has pointed out that in $D=4, N=1$ supersymmetric IIB string orientifold models there is a possibility of obtaining such effective or mirage gauge coupling unification at scales which are much higher than the string scale $[9,10]$. These models generically have $\sigma$ model anomalies in addition to anomalous $U(1)$ gauge groups. All anomalies are cancelled by the Green-Schwarz mechanism which results in moduli dependent corrections to the gauge couplings. When ratio between the coefficients of these corrections ( $\sigma$ model anomaly coefficients) and the $\beta$-functions are constants independent of the gauge group these corrections can be absorbed into the effective unification scale. For models with large compact dimensions the effective unification scale becomes much larger than the string scale. Since there is no running of gauge couplings above $M_{s}$ and there is a fully-fledged string theory at this scale, this unification is a mirage seen from the low-energy point of view. In fact the gauge couplings do not unify at the string scale.

In this letter we show that in TeV scale string scenarios realized by $D=4$, $N=1$ supersymmetric IIB string orientifold models an effective unification of couplings at $M_{U}$ is possible. For this to occur it is important to have an anisotropic compactification such as two large and four string size compact dimensions. Moreover, as we explain below, the $\sigma$ model anomaly coefficients must not all be equal to each other, preferably one much larger than the others. If in addition, these coefficients contain a constant term independent of the gauge group then the well-known relation between the unified gauge coupling and the dilaton $\mathrm{VEV}$ is modified. In this case a string model with intermediate or strong coupling can lead to weakly coupled gauge theories.

\section{Mirage Gauge Coupling Unification}

In this section we consider a simplified model which has all the characteristics of $D=4, N=1$ supersymmetric IIB string orientifold models with large internal dimensions $[11,12,13]$. These models are obtained by compactifying the IIB string 
on $T^{6}=T^{2} \times T^{2} \times T^{2}$ and moding out by world-sheet parity $\Omega$ times a discrete space-time symmetry. The models generically have a chiral spectrum with a large gauge group. Part of the massless spectrum is projected out and the gauge group is broken by Wilson lines. We will assume that a realistic string model can be constructed in this framework even though such a model does not exist yet. Depending on the orientifold group there will be $32 \mathrm{D}$-branes of a given dimension (such as D3 or D5 branes) with 64 orientifold planes with the same dimension. Gauge bosons and matter live on the brane world-volumes whereas gravity only propagates in the bulk. For more details on these models we refer the reader to the refs. $[11,12,13]$. Below we consider a toy model with all the relevant properties of these string models.

For simplicity, we assume that the gauge group is the Standard Model group $S U(3)_{C} \times S U(2)_{L} \times U(1)_{Y}$ and another $U(1)_{X}$ which is anomalous. This anomaly is cancelled by the Green-Schwarz mechanism. The Kahler potential for the dilaton $S$, the untwisted moduli $T_{i}$ and the matter fields $\phi_{r}$ are given by

$$
K\left(S, S^{*}, T_{i}, T_{i}^{*}, \phi_{r}, \phi_{r}^{*}\right)=-\log \left(S+S^{*}\right)-\sum_{i} \log \left(T_{i}+T_{i}^{*}\right)+\sum_{r, i} \frac{\phi_{r} \phi_{r}^{*}}{\left(T_{i}+T_{i}^{*}\right)^{n_{r}^{i}}}
$$

where $n_{r}^{i}$ are the modular weights of the matter fields $\phi_{r}$ with $\Sigma_{i} n_{r}^{i}=1$. The gauge function for the group denoted by index $a(a=1,2,3)$ is given by

$$
f_{a}=S+\frac{\delta_{a}}{2} M
$$

where $M$ is the overall twisted modulus of the model and $\delta_{a}$ is a constant which depends only on the gauge group. This model has four anomalies; the $U(1)_{X}$ anomaly and three $S L(2, R)_{i} \sigma$ model anomalies due to the three $S L(2, R)_{i}$ transformations[10]

$$
T_{i} \rightarrow \frac{a_{i} T_{i}-i b_{i}}{i c_{i} T_{i}+d_{i}}
$$

with $a_{i}, b_{i}, c_{i}, d_{i}$ real and $a_{i} d_{i}-b_{i} c_{i}=1$. All the anomalies are cancelled by the Green-Schwarz mechanism if the imaginary part of the twisted modulus transforms 
as $\left(\Lambda_{X}\right.$ is the $U(1)_{X}$ gauge parameter $)$

$$
\operatorname{Im} M \rightarrow \operatorname{Im} M+\delta_{G S}^{X} \Lambda_{X}
$$

under the $U(1)_{X}$ and as

$$
\operatorname{Im} M \rightarrow \operatorname{Im} M-2 \sum_{i} \delta_{G S}^{i} \log \left(i c T_{i}+d\right)
$$

under the three $S L(2, R)_{i}$ model transformations. Here $\delta_{G S}^{X}$ and $\delta_{G S}^{i}$ are the anomaly coefficients of the $U(1)_{X}$ and $\sigma$ model anomalies respectively. We also assume that the twisted modulus has the Kahler potential

$$
K\left(M, M^{*}\right)=\left(M+M^{*}-\delta_{G S}^{X} V_{X}-\sum_{i} \delta_{G S}^{i} \log \left(T_{i}+T_{i}^{*}\right)\right)^{2}
$$

which is invariant under both $U(1)_{X}$ and the $\sigma$ model transformations. The above Kahler potential induces an anomalous D-term $[9,10]$

$$
\xi_{X}=-\delta_{G S}^{X}\left(M+M^{*}-2 \sum_{i} \delta_{G S}^{i} \log \left(T_{i}+T_{i}^{*}\right)\right)
$$

If we are interested in supersymmetric vacua then $\xi_{X}=0$ which means

$$
R e M=\sum_{i} \delta_{G S}^{i} \log \left(T_{i}+T_{i}^{*}\right)
$$

As a result, the gauge couplings are given by

$$
\frac{8 \pi^{2}}{g_{a}^{2}}=R e S+\frac{1}{2} \sum_{i}\left(\delta_{G S}^{i} \delta_{a}\right) \log \left(T_{i}+T_{i}^{*}\right)
$$

The product of the two anomaly coefficients is $\delta_{G S}^{i} \delta_{a}=b_{a}^{i \prime}$ where[10]

$$
b_{a}^{i \prime}=-C\left(G_{a}\right)+\sum_{R_{a}} T\left(R_{a}\right)\left(1+2 n_{R_{a}}^{i}\right)
$$

Here $C\left(G_{a}\right)$ and $T\left(R_{a}\right)$ are the second Casimir invariants of the adjoint and $R_{a}$ representations of the gauge group $G_{a}$. Moreover, it can be shown that $\Sigma_{i} b_{a}^{i \prime}=$ 
$\beta_{a}$ where $\beta_{a}$ are the $\beta$-functions corresponding to the minimally supersymmetric standard model.

The running gauge couplings become[9]

$$
\frac{8 \pi^{2}}{g_{a}^{2}}=R e S+\frac{1}{2} \sum_{i} b_{a}^{i \prime} \log \left(T_{i}+T_{i}^{*}\right)+\frac{1}{2} \beta_{a} \log \frac{M_{s}^{2}}{Q^{2}}
$$

Here we assume that there are no threshold states including Kaluza-Klein states up to the string scale. This is only possible if the Standard Model degrees of freedom arise from the world-volume of D3 branes so that there are no brane directions wrapped around string size compact dimensions. We see that the correction due to the $\sigma$ model anomaly gives an extra moduli dependent logarithmic term in the running coupling constant. If $b_{a}^{i \prime}=r_{i} \beta_{a}$ with $r_{i}$ a constant independent of the gauge group then we see that the moduli dependent corrections can be combined with $M_{s}$ to give an effective unification scale

$$
M_{X}=M_{s} \prod_{i}\left(T_{i}+T_{i}^{*}\right)^{r_{i} / 2}
$$

The untwisted moduli $T_{i}$ are related to the compactification radii by

$$
2 \operatorname{Re} T_{i}=\frac{4 R_{j}^{2} R_{k}^{2} M_{s}^{4}}{g_{s}}
$$

Therefore we get

$$
M_{X}=\frac{2 M_{s}^{3}}{g^{3 / 2}}\left(R_{j} R_{k}\right)^{r_{i}}\left(R_{i} R_{k}\right)^{r_{j}}\left(R_{i} R_{j}\right)^{r_{k}}
$$

We have found that due to the $\sigma$ model anomaly there is an extra moduli dependent logarithmic term in the running coupling constant. Under certain conditions i.e. $b_{a}^{i \prime}=r_{i} \beta_{a}$ for all gauge groups, this term modifies the unification scale. We see that effectively the unification scale becomes $M_{X}$ with the unified gauge coupling $g_{U}$ given by $R e S$. In models with large compct dimensions the scale $M_{X}$ is 
much larger than the string scale $M_{s}$. However, we know that the gauge couplings do not run above the string scale, $M_{s}$, in fact above $M_{s}$ we have a fully-fledged string theory. Therefore, the field theoretical running of the gauge couplings up to $M_{X}$ and their unification at $M_{X}$ are just mirages seen from the low energies. Note that at the string scale the gauge couplings are not unified but given by eq. (12) with $Q^{2}=M_{s}^{2}$.

In fact we can go further and assume that $b_{a}^{i \prime}=r_{i} \beta_{a}+\eta_{i}$ where $\eta_{i}$ is a constant independent of the gauge group. Then we find that in addition to the modification of the unification scale, there is a constant term which can be absorbed into the dilaton VEV. We have

$$
\frac{8 \pi^{2}}{g_{U}^{2}}=R e S+\sum_{i} \eta_{i} \log \left(T_{i}+T_{i}^{*}\right)
$$

This result is very interesting. If $\eta_{i}=0$ then the unified gauge coupling $g_{U}$ is fixed by $R e S$. In particular since $\alpha_{U}=1 / 24$ we need $\operatorname{Re} S \sim 150$ a large value exactly as in heterotic string theory. Since $\operatorname{Re} S=2 / g_{s}$ this also corresponds to a weakly coupled string theory. However, if $\eta_{i} \neq 0, \operatorname{Re} S$ does not need to correspond to the unified gauge coupling. In particular, it can be much smaller than 150 or even of $O(1)$ depending on $\eta_{i}$ and the size of the compact dimensions. In this case there is the possibility of having weakly coupled gauge interactions in a string theory with intermediate (or strong) i.e. $O(1)$ coupling.

Our aim is to find out whether an effective mirage unification of the gauge couplings can be obtained with $\alpha_{U} \sim 1 / 24$ and $M_{X} \sim 2 \times 10^{16} \mathrm{GeV}$ in this framework. We first assume that $\eta_{i}=0$ and the compactification is isotropic so that there is only one overall untwisted modulus $T=T_{1}+T_{2}+T_{3}$. Then using $\Sigma_{i} b_{a}^{i \prime}=\beta_{a}$ we find

$$
M_{X}=\frac{2 R^{2} M_{s}^{3}}{g_{s}^{3 / 2}}
$$


where the compactification radius $R$ is given by

$$
R^{6}=\frac{g_{s}^{2} M_{P}^{2}}{8 M_{s}^{8}}
$$

As observed in ref. [9] this gives $M_{X} \sim 10^{13} \mathrm{GeV}$ which is three orders of magnitude smaller than the desired value. Of course, this example is too simple because in models with large internal dimensions, the compactification is not isotropic and one should consider the three untwisted moduli $T_{i}$ separately.

We now consider TeV scale strings compactified on two large and four string size dimensions. The cases with more than two large dimensions can be easily generalized from this case and work as well. The size of the large dimensions is given by

$$
R^{2}=\frac{g_{U}^{4} M_{P}^{2}}{32 \pi^{2} M_{s}^{4}}
$$

If the two large dimensions are on the same torus we have

$$
2 R e T_{1}=2 \operatorname{Re} T_{2}=\frac{g_{U}^{2}}{4 \pi}\left(\frac{M_{P}}{M_{s}}\right)^{2}
$$

and

$$
2 R e T_{3}=\frac{8 \pi}{g_{U}^{2}}
$$

We now need to assume values for $b_{a}^{i^{\prime}}$ which need to be proportional to $\beta_{a}$ independently of $a$. We take for example $r_{i}=(3 / 4,1 / 8,1 / 8)$ for the three tori $T_{i}^{2}$. We stress that this choice is the same for the three gauge groups $S U(3)_{C}, S U(2)_{L}, U(1)_{Y}$. Then

$$
M_{X} \sim \alpha_{U}^{5 / 16} M_{P}^{7 / 8} M_{s}^{1 / 8}
$$

For $M_{P} \sim 10^{19} \mathrm{GeV}, M_{s} \sim 50 \mathrm{TeV}$ and $g_{U}^{2} \sim 1 / 2$ we find $M_{X} \sim 2 \times 10^{16} \mathrm{GeV}$ which is the desired unification scale predicted by the minimally supersymmetric 
Standard Model. We see that the dependence on the string scale $M_{s}$ is very weak so that a change of $M_{s}$ between $1 \mathrm{TeV}$ and $50 \mathrm{TeV}$ gives only a factor of $(50)^{1 / 8} \sim 1.5$. This means that mirage unification is not too sensitive to the lowest bound on the string scale, $M_{s}$ or to the distinction between $M_{s}$ and the higher dimensional Planck scale $M_{d+4}$. Also note that for the above choice of $b_{a}^{i \prime}$ either the matter content of the Standard Model arises in an asymmetric manner from the sectors corresponding to the three tori or the modular weights $n_{r}^{i}$ are not the same in these sectors.

The choice above for $b_{a}^{i \prime}$ is a very unique one and it is not clear why the anomaly coefficients should satisfy this. However, we remind that the above holds only for $\eta_{i}=0$. If $\eta_{i} \neq 0$, we can change each one of the anomaly coefficients by a constant which does not depend on the gauge group. Thus, there are many values of $b_{a}^{i^{\prime}}$ that will result in mirage gauge coupling unification. The only requirement on the coefficients $b_{a}^{i^{\prime}}$ seems to be that they be the same for all gauge groups for a given torus.

On the other hand, when $\eta_{i} \neq 0$ the value of the unified gauge coupling does not arise solely from $R e S$ as given in eq. (10). In order to estimate the magnitude of this effect we assume that $\eta_{1} \neq 0$ but $\eta_{2}=\eta_{3}=0$. Then

$$
\frac{8 \pi^{2}}{g_{U}^{2}}=R e S+\eta_{1} \log \left(T_{1}+T_{1}^{*}\right)
$$

Using eq. (20) we find that the correction to $\operatorname{Re} S$ is $\sim 30 \eta_{1}$. So even for $\eta_{1} \sim 1$ this is an important effect. Of course this effect is even larger when all $\eta_{i} \neq 0$. If $\eta_{1} \sim 5, \operatorname{Re} S$ can be of $O(1)$ without changing the unified gauge coupling $g_{U}$. Thus, we find the interesting possibility of getting weakly coupled gauge theories from a string theory which has an intermediate or strong coupling.

\section{Conclusions and Discussion}

In this letter we showed that an effective unification of gauge couplings at $M_{U} \sim 2 \times 10^{16} \mathrm{GeV}$ can be obtained in models with TeV scale strings. In models 
with $\sigma$ model anomalies the expression for the gauge couplings get moduli dependent corrections. If the anomaly coefficients are proportional to the $\beta$-functions independently of the gauge group there is an effective new unification scale. For models with large compact dimensions this effective unification scale is much larger than $M_{s}$. However, at $M_{s}$ there is fully-fledged string theory and therefore gauge couplings do not run above this scale. The field theoretical running of the gauge couplings up to energy scales much larger than $M_{s}$ and their unification at $M_{U}$ is a mirage from the low energy point of view. For simplicity we considered only the case with two large compact dimensions but our results can be easily generalized to the cases with more than two large dimensions.

In order for mirage unification to occur for two large dimensions the anomaly coefficients have to satisfy $b_{a}^{i \prime}=r_{i} \beta_{a}$ with $r_{i}=(3 / 4,1 / 8,1 / 8)$ for all the gauge groups. Therefore either the observable sector must arise in an asymmetric manner from the sectors corresponding to the three tori or the modular weights must be asymmetric among these sectors. This is a unique choice for $b_{a}^{i \prime}$ which is not generic at all. However, unification can be maintained if the anomaly coefficients satisfy the modified relation $b_{a}^{i \prime}=r_{i} \beta_{a}+\eta_{i}$. Thus, there are a large number of choices for $b_{a}^{i \prime}$ which result in mirage unification. It is difficult to say how generic this situation is because there are no realistic $D=4, N=1$ supersymmetric IIB orientifold models. The requirement $r_{i}$ have to satisfy for mirage unification is that they be the same for all gauge groups and not be equal to each other. It seems that demanding mirage gauge coupling unification puts strong constraints on $\mathrm{TeV}$ scale string model building. At this stage, it is hard to say whether these can be easily satisfied.

An intriguing result of the expression $b_{a}^{i \prime}=r_{i} \beta_{a}+\eta_{i}$ is the change in the relation between the value of the unified coupling constant and the dilaton VEV. From eq. (10) we see that for some values of $\eta_{i}$ and large compact dimensions, the gauge couplings can be small even though the string coupling $g_{s}=2 / \operatorname{Re} S$ is large. Therefore a string theory with an intermediate or strong coupling can give rise to perturbative gauge interactions. This may facilitate dilaton stabilization in $\mathrm{TeV}$ 
scale string models since now there is no constraint on the dilaton VEV.

\section{REFERENCES}

1. N. Arkani-Hamed, S. Dimopoulos and G. Dvali, Phys. Rev. Lett. B429 (1998) 263, hep-ph/9803315; I. Antoniadis, N. Arkani-Hamed, S. Dimopoulos and G. Dvali, Phys. Lett. B436, 257 (1998), hep-ph/9804398.

2. I. Antoniadis, Phys. Lett. B246 (1990) 377; J. Lykken, Phys. Rev. D54 (1996) 3693, hep-th/9603133; I. Antoniadis and K. Benakli, Phys. Lett. B326 (1994) 69; I. Antoniadis, K. Benakli and M. Quiros, Nucl. Phys. B331 (1994) 313.

3. N. Arkani-Hamed, S. Dimopoulos and G. Dvali, hep-ph/9807344.

4. K.R. Dienes, E. Dudas and T. Gherghetta, Phys. Lett. B436, 55 (1998) hepph/9803466; R. Sundrum, hep-ph/9805471, hep-ph/9807348; G. Shiu and S.H. Tye, Phys. Rev. D58, 106007 (1998) hep-th/9805157; P.C. Argyres, S. Dimopoulos and J. March-Russell, Phys. Lett. B441, 96 (1998) hepth/9808138; N. Arkani-Hamed, S. Dimopoulos and J. March-Russell, hepth/9809124; A. Donini and S. Rigolin, hep-ph/9901443; Z. Berezhiani and G. Dvali, hep-ph/9811378; Z. Kakushadze, hep-th/9812163, hep-th/9902080; Z. Kakushadze and S.-H.H. Tye, hep-th/9809147; N. Arkani-Hamed, S. Dimopoulos, hep-ph/9811353; N. Arkani-Hamed, S. Dimopoulos, G. Dvali and J. March-Russell, hep-ph/9811448; A. Pomarol and M. Quiros, Phys. Lett. B438, 255 (1998); T.E. Clark and S.T. Love, hep-th/9901103; T. Banks, M. Dine and A. Nelson, hep-th/9903019; I. Antoniadis, K. Benakli and M. Quiros, hep-ph/9905311; G. Shiu, R. Schrock and S.-H. Tye, hepph/9904262; E. Halyo, hep-ph/9904432.

5. G.F. Giudice, R. Rattazzi and J.D. Wells, hep-ph/9811291; T. Han, J.D. Lykken and R. Zhang, hep-ph/9811350; J.L. Hewett, hep-ph/9811356; E.A. Mirabelli, M. Perlstein and M.E. Peskin, hep-ph/9811337; M.L. Graesser, 
hep-ph/9902310; S. Nussinov and R.E. Shrock, hep-ph/9811323; T.G. Rizzo, hep-ph/9901209; hep-ph/9902273; hep-ph/9903475; K. Agashe and N.G. Deshpande, hep-ph/9902263; K. Cheung, hep-ph/9904266; K. Cheung and W. Keung, hep-ph/9903294; M. Masip and A. Pomarol, hep-ph/9902467.

6. K. Benakli, hep-ph/9809582; K. Benakli and S. Davidson, hep-ph/9810280; M. Maggiore and A. Riotto, hep-th/9811089; D.H. Lyth, Phys. Lett. B448 (1999) 191, hep-ph/9810320; N. Kaloper and A. Linde, hep-th/9811141; G. Dvali and S.-H. H. Tye, hep-ph/9812483; K.R. Dienes, E. Dudas, T. Ghergetta, A. Riotto, hep-ph/9809406; N. Arkani-Hamed, S. Dimopoulos, N. Kaloper and J. March-Russell, hep-ph/9903224; hep-ph/9903239; L. Hall and D. Smith, hep-ph/990426; E. Halyo, hep-ph/9901302; hep-ph/9905244; C. Csaki, M. Graesser and J. Terning, hep-ph/9903319; J. Cline, hepph/9904495; A. Riotto, hep-ph/9904485; G. Dvali, hep-ph/9905204.

7. S. Cullen and M. Perelstein, hep-ph/9903422.

8. K.R. Dienes, E. Dudas and T. Gherghetta, hep-ph/9806292; C. Bachas, hepph/9807415; Z. Kakushadze, hep-ph/9811193; I. Antoniadis and C. Bachas, hep-th/9812093; A. Perez-Lorenzana and R. Mohapatra, hep-ph/9904504.

9. L. Ibanez, hep-ph/9505349.

10. L. Ibanez, R. Rabadan and A. Uranga, hep-th/9905098.

11. G. Aldazabal, A. Font, L. Ibanez and G. Violero, hep-th/9804026.

12. L. Ibanez, C. Munoz and S. Rigolin, hep-ph/9812397.

13. L. Ibanez, R. Rabadan and A. Uranga, hep-th/9808139. 